\title{
El rol de los docentes co-formadores en el proceso de prácticas del Profesorado en Historia. ${ }^{1}$
}

\author{
por Verónica Gatti, Marcela Zatti y Martín Céparo \\ Universidad Autónoma de Entre Ríos \\ veronicagatti73@yahoo.com,marceezatti@hotmail.com,martin_ceparo@hotmail.com
}

Recibido: 02/05/2019 - Aceptado: 03/06/2019

\section{Resumen}

En el Profesorado en Historia de la Universidad Autónoma de Entre Ríos se realizan las prácticas docentes en los niveles de enseñanza secundaria y superior, por lo que nos interesa observar, analizar y comprender los procesos que involucran a los/as estudiantes practicantes en la diagramación y preparación de las clases durante su formación inicial, es decir, las decisiones que se toman y son asumidas al momento de planificar las secuencias didácticas en el marco de las cátedras de Didáctica de la Historia y las Ciencias Sociales y el Taller de Acción Educativa. En este sentido, hemos trabajado previamente con las narrativas escritas por estudiantes en las que se reflejan diversas problemáticas a la hora de dar cuenta de la formulación de propuesta didáctica, el manejo de grupo, el posicionamiento del rol docente, entre otras, develando un espectro muy amplio sobre sus experiencias, sacando a la luz fortalezas y debilidades. Con el propósito de complejizar esta línea de indagación, hemos decidido en esta oportunidad, trabajar y analizar narrativas que nos brindan los docentes co-formadores. Mediante una entrevista de aproximación metodológica al campo de estudio, nos introducimos en el punto de vista de actores que tienen un rol importante en el proceso de las prácticas ya que nos brindan información desde su experiencia concreta con nuestros/as practicantes, permitiéndonos rever procesos de enseñanza.

\section{Palabras claves}

docentes co-formadores, prácticas de enseñanza, acompañamiento, practicantes

$$
* * *
$$

\section{The role of co-trainer teachers in the process of teaching practices in History.}

\section{Abstract}

In the teaching career in History of the Autonomous University of Entre Ríos, the teaching practices are carried out at secondary and higher levels, so we are interested in observing, analyzing and understanding the processes that involve the practicing students in the diagramming and preparation of the classes during their initial training, that is, the decisions that are made and are assumed when planning the teaching sequences within the framework of the Didactics of History and Social Sciences and the Educational Action Workshop. In this sense, we have previously worked with the narratives written by students in which various problems are reflected when it comes to giving account of the formulation of didactic proposal, group management, positioning of the teaching role, among others, unveiling a spectrum very broad about their experiences, bringing to light strengths and weaknesses. In order to make this line of inquiry more complex, we have decided in this opportunity to work on and analyze narratives provided by co-trainer teachers. Through an interview of methodological approach to the field of study, we introduce ourselves in the point of view of actors that have an important role in the process of the practices since they give us information from their concrete experience with our practitioners, allowing us to review teaching processes.

\section{Keywords}

co-trainer teachers, teaching practices, accompaniment, practitioners

Clío \& Asociados. La historia enseñada. Enero-Junio 2019 (28) ISSN 2362-3063 (digital), pp. 99-I08 Universidad Nacional del Litoral - Universidad Nacional de La Plata (Santa Fe/La Plata -Argentina) 


\section{Introducción.}

El presente escrito se enmarca en un proyecto de investigación -PIDIN- a desarrollarse en el transcurso de 2019 titulado "Procesos de prácticas de enseñanza reflexivas en los/las estudiantes de Profesorados en Historia durante su formación inicial y su relación con la construcción de secuencias didácticas", aprobado y financiado por la FHAyCS-UADER ${ }^{2}$.

En este contexto, desde las cátedras de Didáctica de la Historia y las Ciencias Sociales y el Taller de Acción Educativa pertenecientes al tercer y cuatro año del Profesorado en Historia y de carácter anual, nos hemos propuesto continuar con la indagación que venimos desarrollando en relación a las prácticas docentes que realizan los/las estudiantes de la mencionada carrera, por lo que nos interesa observar, analizar y comprender los procesos que involucran a los/las estudiantes practicantes en la diagramación y preparación de las clases durante su formación inicial, es decir, las decisiones que se toman y son asumidas al momento de planificar las secuencias didácticas.

De esta manera, tomando como antecedentes el análisis realizado a las narrativas escritas por estos/as estudiantes en las que se ven reflejadas diversas problemáticas a la hora de dar cuenta de sus fortalezas y debilidades (Gatti, Zatti y Céparo, 2018), y con un propósito claro de continuar con esta línea de indagación, hemos decidido en esta oportunidad trabajar y analizar narrativas que nos brindan los docentes co-formadores obtenidas a partir de entrevistas formuladas mediante preguntas guiadas, por lo que la aproximación metodológica en este caso, es de corte cualitativo. La entrevista utilizada de tipo estandarizada abierta se caracteriza por el empleo de un listado de preguntas ordenadas y redactadas de igual manera para todos los entrevistados, pero de respuesta libre o abierta; algunos autores la consideran como una entrevista de investigación- research interview-, una técnica de obtención de información relevante para cumplimentar los objetivos de un estudio (Valles, 2007:180-181).

\section{Experiencias y prácticas de docentes co-formadores.}

El objetivo que guía este trabajo consiste entonces, en recuperar el punto de vista de los docentes co-formadores, puesto que entendemos son actores claves y tienen un rol importante en el proceso de las prácticas al sacar a la superficie situaciones que nos permiten rever los procesos de formación inicial que desarrollan los/las estudiantes practicantes y la práctica docente concreta en los diferentes establecimientos escolares de enseñanza secundaria en la ciudad de Paraná y localidades aledañas.

Si bien cada estudiante practicante cuenta con el acompañamiento del docente especializado de las prácticas, orientando y asesorando pedagógicamente, cobra un papel fundamental el rol del docente co-formador quien durante un período aproximado de dos a seis meses ${ }^{3}$, observa, acompaña, guía diariamente al futuro/a profesor/a en su desempeño áulico en relación al desarrollo de las actividades previamente planificadas.

El docente co-formador orienta en la planificación de las clases, debido a que es el único que conoce el grupo de estudiantes secundarios, presenta una dinámica de trabajo y experiencias que le son propias a cada situación, es además, quién puede solucionar o anticipar alguna problemática acudiendo a estrategias orientadoras que favorezcan la enseñanza, el aprendizaje, la evaluación, el uso del tiempo áulico de trabajo, proponiendo cambios que surjan en la inmediatez de las práctica. Es decir, es el que ayuda, contiene y cede espacios para el ejercicio del proceso de enseñanza y aprendizaje. Asimismo, es el que reconoce las características de la cultura institucional, quienes ocupan los lugares de gestión, facilitando y mediando en la incorporación de los/las practicantes, ya que también nos encontramos con instituciones que no habilitan la incorporación de los mismos.

De esta manera, "el trayecto de la residencia [prácticas docentes en nuestro caso] es entendido como una categoría espacio-temporal en la que se articulan, como una especie de "bisagra", la dimensión subjetiva y la objetiva del proceso de construcción con los otros agentes" (Spakowsky, 2006:47), es decir, nosotros como profesores de las prácticas, los docentes co-formadores, los pares e integrantes de la institución: directivos, docentes en general, estudiantes, administrativos, ordenanzas, etcétera. 
Sin embargo, una problemática importante a atender es la posible influencia que suelen ejercer los docentes co-formadores desde su experiencia e impronta en la toma de decisiones de los/las practicantes. Por esta razón, es importante establecer acuerdos previos respecto a la planificación de la secuencia didáctica y la consulta bibliográfica específica, con el objeto de contemplar y utilizar fuentes, recursos variados, estrategias y actividades que permitan el desarrollo de competencias cognitivas en los/las estudiantes secundarios, al mismo tiempo, considerar las instancias del trabajo de aula, la selección de contenidos, los recortes temporales, los sujetos de la historia, la evaluación, atendiendo y revisando cuatro aspectos que configuran y tensionan las prácticas y operan en conjunto en la construcción metodológica: pensar históricamente (qué), analizar las finalidades de la enseñanza de la historia (para qué y por qué), indagar respecto a la construcción del pensamiento escolar (a quiénes) y, pensar didácticamente (cómo), con el propósito de generar un proceso de reflexión en relación a la toma de decisiones (Cerdá y Jara, 2016).

Por lo mismo, y siguiendo la línea de análisis de Perrenoud (2011), es necesario fortalecer la confianza en cada practicante desarrollando competencias especializadas, conocimientos exhaustivos, capacidad de juicio, de anticipación, de análisis y de innovación. En este sentido, la figura del docente co-formador se muestra como un referente capaz de orientar con recursos necesarios para procurar autonomía y reflexión sobre el propio proceso de prácticas.

Desde esta perspectiva, la elección del docente co-formador se presenta como un desafío para las cátedras de Didáctica de la Historia y las Ciencias Sociales y el Taller de Acción Educativa. Comienza con la selección de las escuelas secundarias, por lo que el primer acercamiento lo realizamos los/las docentes a cargo de las mismas con el fin de solicitar autorización a los equipos directivos y recabar información sobre los docentes, cursos, días, horarios, turnos.

Con esta información y una vez en las respectivas clases, se comienza con la distribución y ubicación de los/las estudiantes practicantes en diferentes turnos (mañana, tarde y noche) según sus disponibilidades y posibilidades. A partir de este momento comienza el contacto con los docentes co-formadores, donde el vínculo y la comunicación se vuelven fluidos y constantes.

Es por esta razón que entendemos que el docente co-formador es un tutor-referente de los procesos de prácticas que realizan los/las estudiantes. Su rol cobra sentido al ser un nexo, un puente entre la formación inicial y la práctica situada en los establecimientos educativos, colaborando de esta manera en la construcción del conocimiento de las prácticas pre-profesionales con miras a la futura inserción laboral.

Por lo antes dicho, el docente co-formador se vuelve un compañero de ruta, para el/la estudiante practicante al cooperar permanentemente en el proceso de formación inicial. Durante el acompañamiento, se comparten diferentes momentos y situaciones cotidianas que permiten visualizar la tarea de enseñar de manera contextuada, situada, singular y hasta impredecible, propias de cada institución escolar, por lo que se constituyen en el referente inmediato y más cercano. Es decir, son quienes al compartir día a día la tarea en el espacio del aula, les permiten vivenciar las prácticas en toda su complejidad, multiplicidad de tarea, imprevisibilidad y singularidad.

Tal como explica Edelstein (2015:151), "la Residencia implica siempre una doble referencia permanente para los sujetos que la realizan" en la que se entrecruzan las instituciones formadoras y las que ofrecen espacios para practicar. De ahí la relevancia de articular criterios entre los docentes formadores con los co-formadores acerca de los modos de acompañar a los/las futuros/as profesores/as, dado que son quienes voluntariamente los aceptan en sus clases con el propósito concreto de que puedan ejercitar la tarea de enseñar un contenido disciplinar. De esta manera se vuelven también, un referente de valoración directo en el espacio del aula.

\section{Docentes co-formadores: una mirada sobre sus prácticas.}

Como se trata de un proyecto que comienza a implementarse, trabajamos en esta oportunidad con tres entrevistas realizadas a docentes co-formadores, a los que nos referiremos con sus iniciales con el objeto de salvaguardar su identidad, por los que los nombraremos como CM, GZ y SU. 
Se trata de tres docentes con recorridos profesionales diferentes. Egresados en distintos años de nuestra casa de estudios, profesores en Historia, con formación de posgrado (finalizada o en proceso) y con experiencia en los niveles secundario y superior. Respecto a la antigüedad, CM cuenta con diecisiete años de servicios y es titular en la mayoría de sus horas en el nivel secundario, desempeñándose como auxiliar y docente interino en el ámbito universitario.

Por su parte, GZ, tiene doce años de antigüedad, siendo titular y suplente en sus horas de secundaria, jefa de trabajos prácticos y docente interina en la universidad. Finalmente SU, cuenta con seis años de antigüedad, es suplente en sus horas de nivel secundario y es también jefa de trabajos prácticos en superior.

En relación a su práctica profesional en las escuelas secundarias, los entrevistados nos cuentan que planifican sus clases, aunque lo hacen de diferente manera. En el caso de SU lo hace más teniendo en cuenta los lineamientos curriculares y proyectos y/o culturas institucionales, mientras que CM lo hace más en función de su experiencia y las expectativas que tiene respecto al grupo de estudiantes. En sus propias palabras

... planifico mis clases en función de la estructura curricular de contenidos, de lineamientos educativos planteados a nivel provincial y nacional, de sugerencias institucionales, vinculándolas con las características de los grupos de estudiantes, desde un enfoque de enseñanza que plantea un abordaje problematizador e interdisciplinario. [SU]

En el nivel secundario planifico anualmente, para todos los niveles, voy viendo como avanzo en todos los niveles. Preparo cada clase pero no las planifico. (...) Y a medida que avanzo veo las necesidades que tienen los estudiantes, cuáles son las dificultades (...). [CM]

... trabajar en la escuela media me dio herramientas de cómo tener planes y desarmarlos frente a la demanda o a la apatía de los estudiantes. [GZ]

Respecto a este punto podemos identificar que en los docentes hay un reconocimiento sobre la necesidad de planificar, pero la diferencia está dada en la trayectoria y antigüedad que posee cada uno. SU plantea una vinculación directa y expresa con los lineamientos curriculares y las cuestiones que surgen de las demandas institucionales sin que ello implique descuidar las que surgen en relación a los grupos estudiantiles, especialmente porque se desempeña en una institución cuya matrícula es de jóvenes y adultos, por lo que la composición etárea muy heterogénea. A su vez, reconoce que su planteo se realiza desde una mirada renovada al focalizar el aspecto problematizador e interdisciplinario de sus planificaciones.

Por su parte, CM es el docente entrevistado con mayor antigüedad, es también, quien indica preparar la clase sin planificarla, en otras palabras, diagrama las clases aunque no las plasma de forma escrita, sino que propone actividades en función de los recursos que selecciona en un dossier de trabajo que propone anualmente. Desde esta lógica, señala que su planificación es anual, haciendo referencia a la documentación exigida por las instituciones educativas con el propósito de cumplimentar con el protocolo y anexar al proyecto educativo de cada escuela. Entonces entendemos que, $\mathrm{CM}$ no vincula la planificación con la organización y diagramación cotidiana de sus clases, por lo que podemos inferir que su propia trayectoria docente le brinda elementos y herramientas para cumplimentar con su trabajo áulico. Aunque sí pone de manifiesto que la enseñanza pasa por la necesidad de atender las necesidades o dificultades que identifica en el estudiantado.

En el último caso, GZ identifica la planificación como una herramienta flexible que puede modificarse en función de las realidades de cada grupo para poder captar la atención o fomentar el trabajo en función de la demanda de los estudiantes secundarios.

Respecto a los recursos didácticos que emplean en sus clases, en los tres casos dan cuenta de textos, fuentes históricas, vídeos y salidas didácticas a diversos espacios (museos, repositorios de archivos, legislatura), entre otros. Su uso está relacionado a las demandas y posibilidades de vinculación con el desarrollo temático y en tanto proponen diferentes estrategias didácticas. SU detalla una gran variedad de propuestas para el abordaje de los recursos mencionados como la 
Exposición oral dialogada, consignas de análisis, observación e interpretación de imágenes, análisis de fuentes, comparaciones, prácticas de lectura en plenario, escritura en el pizarrón, cuadros sinópticos, lluvia de ideas, estudios de caso, preguntas problematizadoras, contraste entre diferentes puntos de vista. [SU]

A su vez, reflexiona sobre la forma en la que reaccionan sus estudiantes secundarios ante la propuesta de clases que consideran actividades y contenidos tendientes a la resolución de situaciones problemáticas y conflictivas.

Depende el grupo, a veces no comprenden las actividades planteadas, otras veces les cuesta realizarlas y otras oportunidades no presentan demasiadas complicaciones al momento de hacerlas y responden bien. Pero en general cuando el grupo es nuevo se resisten a aquellas actividades que impliquen relacionar información o que tenga que ver con resolución de problemas o conflictos, o que tengan que dar una opinión personal. De todas formas aquellos grupos de estudiantes que vienen trabajando bastante tiempo conmigo no tienen dificultades ( excepciones de estudiantes con adaptaciones). [SU]

En el caso de CM señala que como organizador de temáticas y lecturas deja “(...) un dossier de bibliografía para que el alumno saque copia y con eso trabajar en clase, es la base para poder empezar a trabajar." En estos dos fragmentos de la entrevista podemos identificar la impronta docente en relación a la selección previa de recursos. En el primer ejemplo podemos inferir que la puesta en acción de tal variedad de estrategias didácticas enunciadas se condice con la mirada problematizadora con la SU enfoca sus prácticas docentes. En el segundo caso, podemos reconocer una organización más lineal y menos problemática de la enseñanza, donde el recorte propuesto por el docente se encuentra previsto en la selección temática que se organiza en forma de dossier bibliográfico que el/la estudiante debe llevar a las clases para trabajar. Si bien no se descarta otro recurso, el material es considerado "base" para desarrollar las clases.

Es de destacar, siguiendo a Gloria Edelstein (2011) que las prácticas de enseñanza significan básicamente, según los docentes, poner el cuerpo, estar al frente, de frente, enfrente, sostener y sostenerse, exponer y exponerse a otros, rasgo constitutivo del cotidiano escolar. Estas acciones provocan en muchos casos una sensación de fragilidad o vulnerabilidad que se agudiza cuando ingresan al aula presencias no habituales, cuestiones que no fueron consideradas y situaciones impensadas.

\section{La relación del docente co-formador y el practicante de la carrera de Historia.}

Un punto de interés en el presente trabajo es la vinculación/relación que establecen los docentes co-formadores con nuestros estudiantes. Por eso, nos resulta significativo qué dicen y qué piensan sobre las propuestas de clases que se planifican y llevan en el proceso de las prácticas, y al mismo tiempo, cómo observan el desempeño de los/las practicantes.

Aquí debemos considerar que las clases son un ambiente en el que transcurren la vida cotidiana de sus actores y donde se produce sentido a las interacciones en torno al saber. El co-formador debe tener una visión sobre los niveles de significación de una clase escolar. Siguiendo a Marta Souto (2001) sería considerar la vida social de la clase, las relaciones de poder y saber de ella, las paradojas en la comunicación; la vida inconsciente de la clase como espacio intersubjetivo, como campo transferencial y vincular y como red de identificaciones; las clases como espacios tácticos de enseñanza en función de metas y como dispositivos técnicos. En este sentido recuperamos algunas intervenciones de las entrevistas que reflejan algunos de los aspectos mencionados: "En el desarrollo de las planificaciones los practicantes tienen un buen desempeño en general, aceptan apreciaciones y sugerencias que se les van haciendo sobre la marcha de sus propuestas didácticas, asimismo en relación al manejo del grupo y en vinculación al manejo de contenidos”. [SU] 
[La planificación] Es buena, casi todos los chicos que he tenido se han adaptado, tanto en las escuelas nocturnas y en la secundaria diurna. Yo tengo un lugar muy cercano con los practicantes, a veces están cursando en paralelo mis materias, entonces uno puede hablar, comentar, sugerirle cosas como docente del aula. [CM]

Las planificaciones tienen diferentes niveles de complejidad en un primer momento pensar el recorte de contenidos les resulta difícil debido a que deben manejar conocimiento sobre los temas-problemas a trabajar. Otro de los momentos es cómo presentan el trabajo de ideas previas y a lo largo de la secuencia la coherencia con el aporte teórico-metodológico con las actividades en las clases. Por último, la evaluación les cuesta al momento de definir criterios e instrumentos. [GZ]

En las entrevistas de SU y CM podemos advertir la apertura y permeabilidad de los/las practicantes al momento de recibir comentarios y sugerencias por parte de los docentes coformadores con miras a posicionarse frente al grupo de estudiantes y permitirles un mejor desarrollo de su propuesta. Además, CM indica que el hecho de tener estudiantes cursando materias en el nivel superior, le permite establecer otro tipo de vínculos para poder profundizar los comentarios. Por su parte, GZ reconoce las dificultades en el proceso de planificar, particularmente señala la necesaria articulación interna que debe poseer una propuesta didáctica resaltando la instancia de selección de criterios de evaluación y de instrumentos acordes como un nudo problemático en su relación con los/las practicantes.

En cuanto al desarrollo de las propuestas y del uso de los recursos didácticos de los/las practicantes, encontramos que hay una valoración positiva de las propuestas que se distancian de un enfoque que podríamos llamar tradicional ${ }^{4}$, por lo que las actividades cobran una mayor relevancia en tanto motivan y problematizan la enseñanza y por ende, los aprendizajes de los/las estudiantes secundarios/as.

... observo diferencias cuando las clases son más dinámicas en el sentido que se van implementando recursos, metodologías, trabajos, estrategias que implican la complejización de la enseñanza y no son tan expositivas, ya que los estudiantes secundarios se distraen fácilmente $o$ se duermen y es necesario proponerles actividades que los movilicen a involucrarse en la clase. [SU]

En esta mirada, CM sostiene que

Usan recursos variados y muy útiles. El texto es algo básico, no deja de aparecer, después hay un uso de mapas e imágenes que incluso les lleva a plantearles herramientas de interpretación para cada caso y eso está bueno porque no se quedan solo con el dossier de los chicos, lo complementan con otros recursos.

En este aspecto, CM pondera las propuestas que no solo emplean el dossier con el que trabajan sus estudiantes, el cual es utilizado por los/las practicantes durante el proceso de planificación ya que es un material de consulta obligatoria para sus estudiantes y en ese sentido, reconoce que el texto es algo que permanece presente, pero que se complementa con una amplia variedad de recursos que permiten interpretarlo desde otras vías de acceso.

Por su parte, GZ señala que "se observan cambios en la utilización de recursos, existe un mayor apoyo en lo audiovisual, aunque falta práctica en cómo explotarlos". Aquí, podemos reconocer la impronta que incorporan algunos/as practicantes en el uso de materiales que resultan novedosos o que requieren un trabajo de edición o preparación adicional (particularmente con los videos descargados de internet). Este recurso suele incorporarse con un escaso tratamiento y con la idea que solamente sea visualizado, sin mayor actividad posterior, por lo cual en el fragmento de la entrevista se remarca la falta de práctica en relación a su implementación didáctica.

En otro pasaje de las entrevistas nos encontramos con lo que los docentes co-formadores observan como debilidades o dificultades vinculadas al proceso de las prácticas. En algunos casos, 
se observan menciones relacionados con cuestiones intrínsecas y propias de la práctica en sí, tensión, incertidumbre, presión, vulnerabilidad, dado en que la mayoría de los/las estudiantes se trata de la primera experiencia situada en el aula ${ }^{5}$. Es decir, aquellas cuestiones en torno al manejo de grupo, inseguridades en el desarrollo de la secuencia planificada pero al mismo tiempo, aparecen otras que tienen que ver con la formación profesional, los recorridos que queda practicante realiza en la carrera y hasta los compromisos y responsabilidades que entran en juego.

Veamos qué nos indican los docentes co-formadores respecto a un primer aspecto en relación a las debilidades o dificultades:

... algunas debilidades que después se van subsanando a lo largo de las clases, es el manejo de grupos, cuesta que se vean como docentes y organizarlos. Otra dificultad es que entienda cómo rearmar la planificación, tiene que saber cómo moverse y decidir sobre ella, tiene que venir sabiendo qué puede hacer con eso que planificó. [CM]

En otro punto, cabe destacar que también los compromisos y responsabilidades se vinculan con las propias necesidades particulares y personales de cada practicante que impide observar sus recorridos desde una mirada autocrítica y situada en el contexto de las prácticas, por eso SU nos manifiesta que

Las debilidades que puedo reconocer en los practicantes del TAE, en algunos casos, les cuesta buscar recursos didácticos acordes a la propuesta de enseñanza, el apuro por terminar las planificaciones para aprobar sus clases sin detenerse demasiado para repensarlas, la entrega de planificaciones muy cerca del momento de dar las clases y el no cumplimiento de las instancias de observación cuando la escuela está abocada a jornadas institucionales (actos, charlas, entre otras). [SU]

Los otros docentes entrevistados ponen acento en cuanto al manejo de contenidos específicos por parte de los/las practicantes:

El manejo de contenidos específicos es bueno, pero hay chicos que dieron alguna materia hace años y que al no tenerla aprobada hay ciertas cuestiones que aparecen como inseguras. Tampoco quiero decir que porque la aprobó la maneja, pero al estar en cuarto [año] deberían ser más seguros. Si lo veo, una vez que terminan la clase, se los digo o les sugiero bibliografía para que revisen. $[\mathrm{CM}]$

"Me interesa resaltar la falta de manejo de contenidos en las planificaciones como debilidad". [GZ] En este caso también nos encontramos con que las dificultades detectadas están vinculadas a una formación que podríamos considerar endeble, que no brinda seguridad al momento de llevar adelante el proceso de prácticas, asimismo la actitud del docente co-formador, recomendando bibliografía y apostando a la mejora en la formación del/la futuro/a docente.

Igual comentario remarca GZ en relación a la falta del manejo o manejo superficial de los contenidos al momento de desarrollar la clase. Por su parte, y en este contexto de falencias y dificultades, los docentes co-formadores también señalan sugerencias a los/las practicantes. Estas recomendaciones se vinculan con sus recorridos, experiencias y conocimiento de las realidades institucionales en las que se desenvuelven cotidianamente.

La sugerencia que habitualmente les doy es que piensen el trayecto de prácticas educativas como una instancia de aprendizaje, con desafios, frustraciones, gratificaciones $y$ particularidades que acontecen entre el diseño de la planificación y la puesta en escena, ya que las mismas constituyen una etapa en la que se van asimilando distintos aspectos a tener en cuenta en la futura tarea docente, además sirven para replantearse la práctica docente desnaturalizando la idea de que no pueden hacer más de lo que han hecho para mejorar sus prácticas educativas. [SU] 
El aporte que realiza SU desde el lugar de co-formadora es sumamente interesante al plantearles el trayecto de prácticas a los/las estudiantes como un desafío, una apertura a seguir aprendiendo, de seguir formándose, especialmente si consideramos estas instancias de formación inicial dentro de otras posibles y futuras. También es importante destacar que significa a las prácticas como momentos de formación atravesados por frustraciones y gratificaciones, pero fundamentalmente remarcamos la necesidad de interpelar al/la estudiante respecto a que pueden hacer algo más y mejor de lo que están logrando.

"Es importante que en sus prácticas puedan tener las instancias de socialización de las experiencias, ya que allí se pueden producir intercambios que generen más preguntas, mejorar las estrategias metodológicas, y también la creatividad en la/os estudiantes". [GZ] En este pasaje de la entrevista y en relación a las sugerencias, GZ trae sobre el tapete dos cuestiones centrales y complementarias. Una, la necesidad de socializar los procesos de prácticas con sus debilidades y fortalezas apostando al aprendizaje colaborativo y dos, nada mejor que para lograr el intercambio de experiencias favorecer la dinámica de talleres o plenarios de autoevaluación, tal como se propone desde Didáctica de la Historia y las Ciencias Sociales y el Taller de Acción Educativa.

En cuanto a las recomendaciones que realiza CM, están más relacionadas con el trabajo cotidiano en el contexto de la clase, con miras de centrar la atención en la puesta en escenas de la enseñanza misma. En tal sentido nos indicaba que

Les digo cómo es el curso, que material podrían trabajar, alguna sugerencia de actividad o recurso. Asesorarlo donde puedo ayudar, sin meterme en el lugar de los docentes que en definitiva lo tienen que evaluar. Siempre le sugiero algo, enfócate en tal cosa, mirá como responden a esto y así después que termine la clase, para no interrumpirle y ponerlo nervioso durante la clase. [CM]

También es importante recuperar las fortalezas que los co-formadores destacan en relación a las prácticas docentes, dado que junto a las recomendaciones se constituyen en momentos de fortalecimiento y empoderamiento para los/las estudiantes, más aún si tenemos en cuenta que al desempeñarse como practicante, lo hacen desde un rol docente enfrentando una doble paradoja, por un lado, ejercer la profesión sin estar recibidos y, por el otro, estar aprendiendo a dar clases al mismo tiempo que son observados y evaluados por lo que y siguiendo a Akcan y Tatar (2010), necesitan consolidar su propia reflexión y autonomía.

En el relato de SU y, a diferencias de CM y GZ, destaca como fortaleza la formación de los/las estudiantes del Profesorado. Evidentemente el comentario surge en relación a su experiencia en torno al desempeño de los/las practicantes, porque como se señala en el párrafo que sigue, destaca una marcada diferencia entre quienes son nuestros estudiantes y aquellos que pertenecen a otras instituciones educativas, al mismo tiempo recupera la capacidad de escucha que tienen respecto a las sugerencias que se les plantean.

Las fortalezas tienen que ver con el nivel de formación y su predisposición para escuchar sugerencias o comentarios inherentes al diseño de sus planificaciones y el desarrollo de sus prácticas. (...) He tenido practicantes provenientes de instituciones de Nivel Terciario, las experiencias no han sido cómodas en tanto el nivel de formación docente es notoriamente inferior a los practicantes de la FHAyCS. Además, no demuestran interés por replantear sus prácticas docentes. [SU]

Este último dato nos resulta relevante y significativo para la tarea que realizamos como docentes a cargo de la formación inicial en el profesorado en Historia. De los tres docentes co-formadores entrevistados, SU es la única que comenta tener experiencia con otros procesos de prácticas, las cuales confronta con las que realizan nuestro/as estudiantes con un saldo positivo a favor de quienes los desempeñamos y acompañamos.

A manera de cierre provisorio, podemos señalar considerando los aportes de Hermida, Pionetti y Segretin (2017) que el ejercicio de narrar las observaciones mediante el análisis de las entrevistas aplicadas a docentes co-formadores, nos permiten visualizar el acompañamiento y tutoría que 
realizan junto a los/las estudiantes practicantes, ayudándolos a reflexionar, confirmar o reformular sus diseños de clases o estrategias implementadas, indicando y observando en la mayoría de los casos, situaciones de mejoras, logros, progresos, proponiendo al mismo tiempo, ajustes y recomendaciones necesarias. es decir, nos permiten recuperar el valor de las escenas o tensiones producidas en el escenario del aula, entrecruzando nuestra práctica docente formadora en el grado, la que ocurre en las instituciones en donde se desempeñan los docentes co-formadores, las que llevan a cabo los/las estudiantes practicantes, posibilitando futuras instancias de investigación, capacitación y transferencia a otros niveles.

\section{Reflexiones y conclusiones.}

Con este trabajo pretendemos aportar un análisis más a la línea de investigación que tiene como objeto de estudio a las prácticas de enseñanza, situación que nos interpela como docentes formadores en el grado. De ahí que resulte relevante articular con la mirada e interpretación que tienen los docentes co-formadores acerca del acompañamiento que realizan a los/las estudiantes practicantes, como así también valorar, especialmente en las instancias de taller, sus recomendaciones y sugerencias en pos de lograr una mejora en las prácticas, dado que son actores claves y decisivos al ejercer un rol de tutor u orientador en el desarrollo de todo el proceso. Siguiendo a Gloria Edelstein (2011), el análisis de las prácticas debe contemplar diversos momentos sobre cómo pensar la clase, construir el diseño, trabajar en y con la inmediatez, recuperar las memorias de experiencias y objetivaciones, para luego problematizar y reescribir nuevamente la clase.

Más allá de los aspectos que se señalan como problemáticos y que deben mejorarse y superarse, nos detenemos en la valoración positiva que presentan los docentes co-formadores en cuanto a las propuestas que se realizan desde la instancia de formación inicial, las cuales se distancian de los que podemos denominar un enfoque o enseñanza tradicional de la historia, tal como lo conceptualiza Rivas (2017). Desde esta valoración se considera que las propuestas de clases atienden a las características de los grupos de estudiantes secundarios, como asimismo a las posibles dificultades que se puedan presentar, por lo que adquieren un formato flexible y permeable a la revisión y/o modificación en función de las realidades de cada grupo o a las demandas institucionales.

Otro aspecto que se valora tiene que ver con la utilización de los recursos didácticos, cuya demanda se incrementa en función de su diversidad con el claro propósito de captar la atención de los/las estudiantes. Entonces, una cuestión clave que surge en las entrevistas está referida al uso de los espacios áulico o de otros posibles espacios fuera del aula, al compromiso de enseñar una historia más compleja y totalizadora desde diversas perspectivas e incorporando nuevos objetos de estudio, de allí la importancia de la interdisciplinariedad y la multiplicidad de fuentes. Al mismo tiempo, se pondera la apertura y permeabilidad de los/las practicantes al momento de recibir comentarios y sugerencias por parte de los docentes co-formadores.

Por su parte y en relación a las dificultades, algunas de ellas están relacionadas al manejo de grupo, inseguridades en el desarrollo de las secuencias planificadas o al uso didáctico poco adecuado de algunos recursos o dispositivos tecnológicos. Lo que sí resulta significativo, son las dificultades que aparecen en relación a la formación profesional, a los recorridos que cada practicante realiza en la carrera y hasta los compromisos y responsabilidades que entran en juego. Sin duda que esto último tiene que ver por un lado, con el compromiso que adquiere el/la practicante como futuro profesional y por otro, con posibles desafíos que se presenten en relación a la innovación educativa.

Finalmente, creemos que este tipo de análisis, reflexiones e intervenciones respecto a la investigación de las prácticas en la formación inicial pueden complementarse con registros de observaciones, de entrevistas que den la palabra a otros actores, como pueden ser estudiantes, directivos o personas del ámbito académico. 
Asimismo, consideramos que reconocer la influencia y los aportes de los docentes co-formadores abre la necesidad de trabajar, desde la Universidad una instancia de complementariedad con el fin de recuperar, valorizar y problematizar las prácticas áulicas. Las entrevistas realizadas implican una mirada y reescritura de la clase, que siguiendo a Edelstein (2011), se plantean como un retorno reflexivo, que vuelve sobre el docente, como memoria de experiencia, recuperación de tramas de sentidos tanto en la formación inicial como continua.

\section{Bibliografía}

Akcan, S. \& Tatar, S. (2010). An investigation of the nature of feedback given to preservice English teachers during their practice teaching experience. Teacher Development, 14(2), 153-172.

Cerdá, ᄃ. \& Jara, M. A. (comp.) (2016). La enseñanza de la Histaria, ante los desafías del bicentenario de la independencia. Córdaba: APEHUN/Pueblo de la Toma.

Edelstein, G. (2011). Formar y formarse en la enseñanza. Buenos Aires: Paidós.

Edelstein, G. (2015). La enseñanza en la farmación para la práctica. Educación, Formación e Investigación, l(1), s/d. Recuperada de http://ppct.caicyt.gov.ar/index.php/efi/article/view/6219/5662

Gatti, V.; Zatti, M. \& Céparo, M. (2018). Los recorridas de las Prácticas Docentes: una autnevaluación a partir de las narrativas de los estudiantes del profesarada de Historia. XVII Larnadas Nacionales y VI Internacionales de Enseñanza de la Historia; II Jarnadas Nacianales Red de Dacentes e Investigadares en la Enseñanza de la Geagrafía y IV Encuentru Iberaamericana de Investigación en Didáctica de las Liencias Saciales, San Carlas de Barilache: UNCa.

Hermida, C.; Pionetti, M. \& Segretin, L. (2017). Formación dacente y narración, Buenos Aires: Noveduc.

Perrenoud, P. (2011). Desarrallar la práctica reflexiva en e/ aficia de enseñar. Barcelona: Graó.

Rivas, A. (2017). Cambia e innavación educativa: las cuestiones cruciales, Buenos Aires: Santillana.

Souto, M. (2001). La clase escolar. Una mirada desde la didáctica grupal. En Camillani, A. R. W. de; Davini, M. C.; Edelstein, G.; Litwin, E.; Souta, M. \& Barco, S. Carrientes didácticas cantemporáneas (119-155). Buenos Aires: Paidós.

Spakawsky, E. (2006). Formación dacente y construcción de la identidad profesional. En Malajovich, A. (comp.). Experiencias y reflexiones sabre la educación inicial: una mirada latinaamericana (211-238). Buenos Aires: Siglo XXI.

Valles, M. S. (2007). Técnicas cualitativas de investigación sacial. Reflexión metadalógica y práctica profesianal. Madrid: Síntesis.

\footnotetext{
Notas

${ }^{1}$ Una versión preliminar fue presentada en la mesa "Enseñar y aprender historia: tensiones y desafíos" del VIII Congreso Regional de Historia e Historiografía, los días 30 y 31 de mayo en la Universidad Nacional del Litoral, Santa Fe, Argentina.

${ }^{2}$ Directora: Verónica Gatti y codirectora: Marcela Zatti. Equipo de investigación: Martín Céparo, Georgina Zárate y Sonia Unrein. Investigadora externa: Mariela Coudannes.

${ }^{3}$ El período de tiempo en el que cada practicante permanece en las instituciones queda sujeto a diversas situaciones contextuales y objetivas propias de cada establecimiento educativo, por lo que si bien todos/as los/las estudiantes realizan la misma cantidad de prácticas, la estadía es relativamente diferente y singular.

${ }^{4}$ Entendemos por enseñanza tradicional siguiendo a Rivas aquella que se rige por pautas establecidas de antemano en las que el currículo propone un ordenamiento y jerarquización de los contenidos alejados de la realidad, lo que trae aparejado dos cuestiones centrales: por un lado, los docentes vuelven rutinarias, poco atractivas e interesantes sus clases y por el otro, los estudiantes no se sienten atraídos por lo que se enseña en forma expositiva y repetitiva deviniendo en "conocimiento inerte" que no genera el deseo de seguir estudiando (Rivas, 2017:26).

${ }^{5}$ En muy pocos casos los/las estudiantes practicantes cuentan con la experiencia de haber dado clases previamente a partir de suplencias, lo que les permite haber ingresado a las instituciones educativas aunque sea en una instancia corta y esporádica.
} 